\title{
Spike timing dependent plasticity promotes synchrony in inhibitory networks in presence of heterogeneity and noise Sachin S Talathi*1,2 and Julie Haas²
}

\author{
Address: ${ }^{1}$ Department of Biomedical Engineering, University of Florida, Gainesville, FL, 32611, USA and ${ }^{2}$ Institute for Nonlinear Sciences, \\ University of California San Diego, San Diego, CA, 92093, USA \\ Email: Sachin S Talathi* - stalathi@bme.ufl.edu \\ * Corresponding author
}

from Sixteenth Annual Computational Neuroscience Meeting: CNS*2007

Toronto, Canada. $7-12$ July 2007

Published: 6 July 2007

BMC Neuroscience 2007, 8(Suppl 2):P58 doi:10.1 I86/I47|-2202-8-S2-P58

(c) 2007 Talathi and Haas; licensee BioMed Central Ltd.

\section{Introduction}

Recently a novel form of spike timing dependent plasticity (STDP) was observed in GABAergic synaptic couplings in layer II of the entorhinal cortex. Depending on the relative timing of pre-synaptic input at time $t_{\text {pre }}$ and postsynaptic excitation at time $t_{\text {post }}$ the synapse is strengthened $(\Delta t=$ $\left.\mathrm{t}_{\text {post }} \mathrm{t}_{\mathrm{pre}}>0\right)$ or weakened $(\Delta \mathrm{t}<0)$. Because the observed effect is the largest at $\pm 10 \mathrm{~ms}$, the operational dynamic range of the observed STDP rule lies in the higher gamma frequency band $(>40 \mathrm{~Hz})$, a frequency range important for several vital neuronal tasks. In this work we study the influence of this novel STDP of inhibitory synapses on the synchronization of two mutually coupled interneurons (MCI) in the presence of heterogeneity and noise. We demonstrate analytically how this synchronization is brought about by defining the spike response curve (SRC), which measures the nonlinear response of neuron to pre-synaptic input. We present simulation results to demonstrate how the unique features of the STDP increase the robustness of synchronization even in the presence of heterogeneity and noise.

\section{Results}

See Figure 1.

\section{Conclusion}

STDP of inhibitory synapses promotes synchrony between two mutually coupled interneurons thereby making it more robust against intrinsic heterogeneity in firing frequency of the coupled neurons. 
a

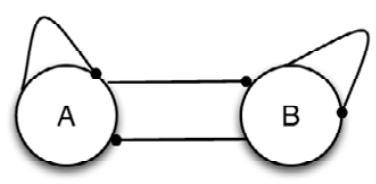

b

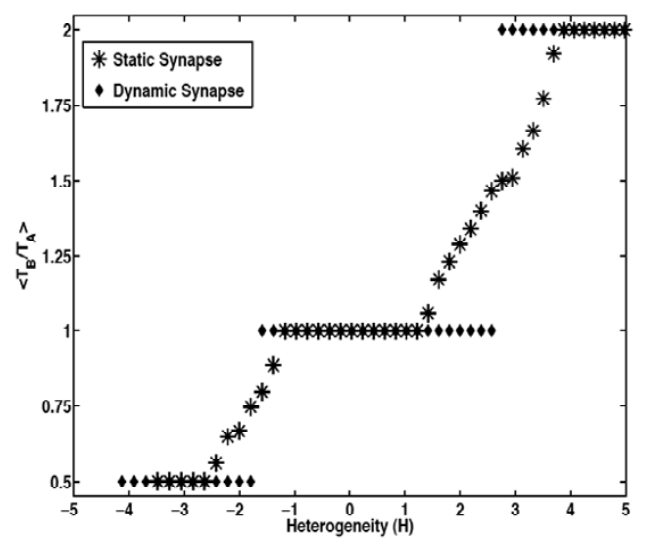

C

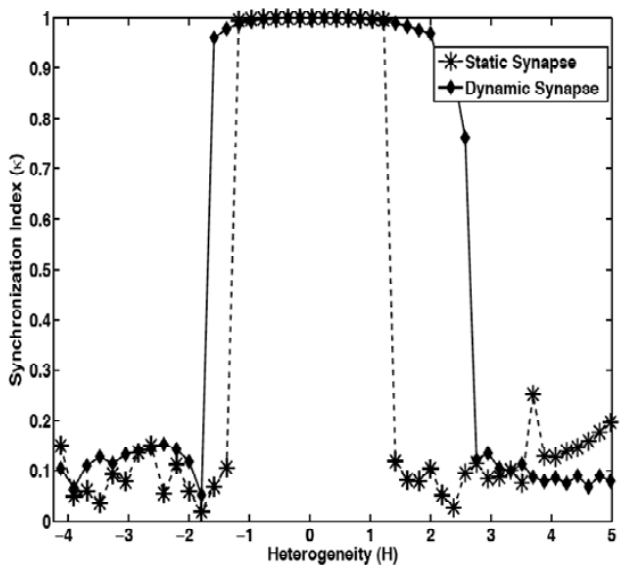

\section{Figure I}

Example demonstrating the enhancement in synchronization of mutually coupled interneurons through spike timing dependent plasticity on inhibitory synapses. (a) Schematic diagram of reciprocally connected interneurons with self-inhibition. (b) The ratio of average firing period of the two neurons is plotted as function of heterogeneity in intrinsic firing frequency of each neuron. The ratio (diamond) represents a dynamic synapse, where STDP modulates the synaptic strength between the coupled neurons. The ratio in (star) represents the situation when the synaptic strength is static. (c) The synchronization index $\kappa$ is plotted as function of heterogeneity $\mathrm{H}$ for the two cases discussed in (b). 